\title{
Cognitive Stimulation Therapy for Dementia
}

Harleen Rai, MSc ${ }^{a, *}$, Lauren Yates, BSc, PhD ${ }^{b}$, Martin Orrell, PhD, FRCPsych ${ }^{\mathrm{C}} \quad \mathbf{Q 2}$

\section{KEYWORDS}

- Dementia $\bullet$ Cognitive stimulation therapy $\bullet$ CST $\bullet$ Psychological treatment

- Psychosocial intervention • Cognition • Quality of life • Well-being

\section{KEY POINTS}

- Cognitive stimulation therapy is a psychological treatment for people with mild and moderate dementia.

- It is offered in both a group and individual format showing various benefits on cognitive functioning, quality of life, and quality of the caregiving relationship.

- The intervention provides a fun and meaningful approach toward staying mentally stimulated and engaged.

- The World Alzheimer's Report 2014 recommends cognitive stimulation therapy to be offered routinely to people with dementia around the world.

- In the future, cognitive stimulation therapy based approaches will hopefully grow and be made available to people who want and need it the most.

\section{INTRODUCTION}

Cognitive stimulation therapy (CST) is a brief psychological treatment for people with mild to moderate dementia. It offers a person-based approach to help people with de- Q6 mentia to stay mentally stimulated and engaged while providing an optimal learning environment. Over the course of 20 years, CST has grown to be widely used with 3 CST manuals published to date. Currently, CST is the only nonpharmacologic therapy

\footnotetext{
Disclosure Statement: Royalties from the sale of the Making a Difference manuals go to the support of the international CST center at UCL run by Dr Aimee Spector. H. Rai is working with Eumedianet on the development of a web app version of CST as part of her PhD.

${ }^{a}$ Division of Psychiatry and Applied Psychology, Institute of Mental Health, School of Medicine, University of Nottingham, Jubilee Campus, Triumph Road, Nottingham NG7 2TU, UK; ${ }^{\text {b }}$ Division of Psychiatry and Applied Psychology, Institute of Mental Health, School of Medicine, University of Nottingham, Jubilee Campus, University of Nottingham Innovation Park, Triumph

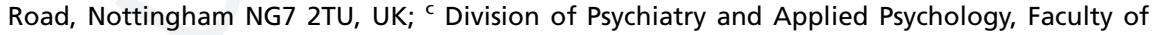
Medicine and Health Sciences, Institute of Mental Health, University of Nottingham, Jubilee Campus, University of Nottingham Innovation Park, Triumph Road, Nottingham NG7 2TU, UK

* Corresponding author.

E-mail address: Harleen.Rai@nottingham.ac.uk
} 
recommended by the National Institute for Health \& Clinical Excellence guidelines (2006) for treating cognitive symptoms of dementia in the UK. These guidelines advise that CST should be available to people with dementia regardless of medication received. In addition, nearly all memory services in the UK currently offer CST in regular groups with people with dementia. ${ }^{1}$ On a global level, CST is now recommended to be offered routinely to people with dementia around the world in the World Alzheimer's Report produced by Alzheimer Disease International in 2011. The International CST Center at University College London (UCL) has supported the adaptation and/or implementation of CST in more than 25 countries. Furthermore, the first 2 international CST conferences brought together researchers, clinicians, and other stakeholders from around the world to discuss past work and to exchange new and exciting ideas regarding CST. In Hong Kong, delegates learned about the concept of a virtual feature for CST groups where people would be able to attend CST groups from the comfort of their homes, connecting with others via a video/audio channel on a technological device. CST shows measurable benefits on cognition and quality of life (QoL) comparable with the effects of some antidementia mediation. In addition, it is cost effective ${ }^{2}$ and very much enjoyed by people with dementia. All of these factors have undoubtedly supported the national and international uptake of the intervention.

The field of CST remains ever evolving and further to what has been achieved so far, there is much to look forward to in terms of innovations.

\section{BACKGROUND}

CST was developed 20 years ago at a time when there were few psychological therapies available for people with dementia and the potential for engagement in mentally stimulating, enjoyable activities in everyday life to preserve cognitive health and protect against decline had not been realized. From the perspective of the population, there was a clear need to have something available that would provide people with dementia with a meaningful way to spend their time. Clinicians and policymakers anticipated the development of new antidementia medication because the benefits of tacrine, the only pharmacologic therapy available, were modest and the risks of adverse events made the drug unsuitable for some people with dementia. Therefore, the field of psychological treatments remained unexplored and trials for psychological interventions were often small in scale and methodologically unsound. From a research perspective, the need for more rigorous investigation of new and/or existing psychological therapies for people with dementia was evident. Considering both the gaps in research and the needs of people with dementia, a research team in the UK set out to develop a novel, psychological therapy whose evaluation would be built on a strong methodological foundation comparable with that of pharmacologic treatments. $^{3}$

The first steps toward developing CST included the review of evidence from existing psychological therapies which could serve as a strong foundation. This review included 2 systematic literature reviews on reality orientation $(\mathrm{RO})$ and reminiscence therapy, 2 widely used psychological approaches. In addition, the work on CST was influenced by Breuil's approach to cognitive stimulation. ${ }^{4}$ Whereas $\mathrm{RO}$ is described as the presentation and repetition of orientation-based information, Breuil's approach differed from traditional RO by setting out to engage people in enjoyable cognitive tasks provided in a group format. Breuil and colleagues $(1994)^{4}$ conducted a randomized, controlled trial among 56 people with dementia and found their cognitive stimulation approach had positive effects on cognitive functioning. The workgroup went on to combine the effective techniques from key therapies $(\mathrm{RO}$, reminiscence therapy 
and, Breuil and colleagues's work) and multisensory stimulation to form the CST program.

CST consists of 14 twice-weekly group sessions (Table 1) that take place over the course of 7 weeks. ${ }^{5}$ All sessions are diverse in nature and the program offers a wide array of topics to ensure it meets the group's interests and cognitive abilities. Every CST group has personalized elements to it, such as choice of a group name and song. These are displayed on an RO board during the session. Sessions last $45 \mathrm{mi}-$ nutes including a 10-minute noncognitive warmup and a 10-minute closing activity (summary and/or the group song). CST is typically delivered by a trained health care professional or care assistant to groups of 5 to 8 people. The facilitators are encouraged to adhere to the key principles of CST, which helps to create the most optimal environment for mental stimulation and enjoyment. Examples of the 18 key principles are mental stimulation, using reminiscence as an aid to the here and now, implicit learning, fun, choice, building/strengthening relationships, and focusing on opinions, rather than facts. These features are unique to CST.

\section{EVIDENCE}

The development of CST followed the guidance of the Medical Research Council framework for developing complex interventions. ${ }^{6}$ This framework includes a development-evaluation-implementation process in which all the phases interact with each other.

The first draft version of CST was taken forward in a pilot study. ${ }^{7} \mathrm{~A}$ total of 27 people with dementia, recruited from a day center and 3 residential homes, were included. Seventeen were randomized to the treatment group receiving CST and 10 were allocated to a treatment as usual control group. The results were promising and indicated that for the CST treatment group there were positive signs regarding cognition, and depression and anxiety seemed to be reduced compared with the control group.

\begin{tabular}{|ll|}
\hline $\begin{array}{l}\text { Table } 1 \\
\text { Cognitive stimulation therapy sessions }\end{array}$ \\
Session & Content \\
\hline 1 & Physical games \\
\hline 2 & Sound \\
\hline 3 & Childhood \\
\hline 4 & Food \\
\hline 5 & Current affairs \\
\hline 6 & Faces/scenes \\
\hline 7 & Associated words \\
\hline 8 & Being creative \\
\hline 9 & Categorizing objects \\
\hline 10 & Orientation \\
\hline 11 & Using money \\
\hline 12 & Number games \\
\hline 13 & Word games \\
\hline 14 & Team games \\
\hline
\end{tabular}

Data from Spector A. Introduction. In: Yates LA, Yates J, Orrell M, et al, editors. Cognitive stimulation therapy for dementia: history, evolution and internationalism. 1st edition. Oxford (England): Routledge; 2017. p. 177-93. 
No negative effects were observed as a result of the treatment. The positive findings from this pilot study formed a strong argument for investigating the effects of CST in a large randomized, controlled trial.

After a few adjustments to the CST program according to the findings from the pilot study, a single-blind, multicenter randomized, controlled trial was conducted that included 201 people with dementia. ${ }^{8}$ The participants were distributed over 23 CST groups and were recruited from 5 day centers and 18 care homes. The following inclusion criteria applied to all participants:

- Diagnostic and Statistical Manual of Mental Disorders, fourth edition, criteria for dementia $^{9}$;

- Score of between 10 and 24 on the Mini Mental State Examination ${ }^{10}$;

- Some ability to communicate and understand (eg, ability to give informed consent);

- Able to see and hear well enough to participate in the group and make use of most of the material in the program; and

- No major physical illness, learning disability, or other disability that could affect participation.

These inclusion criteria have been commonly applied in CST studies since, and are now referred to as the Spector and colleagues (2003) standardized criteria.

Participants were randomized to either a CST group $(n=115)$ or a treatment as usual control group $(n=86)$. Researchers aimed to assess benefits across several outcomes measures with primary outcomes of cognitive functioning and $\mathrm{QoL}$. The trial results were positive: participants in the CST group showed significant improvements in cognitive functioning as measured by the Mini Mental State Examination ${ }^{10}$ and the Alzheimer's Disease Assessment Scale Cognitive Subscale (ADAS-Cog) ${ }^{11}$ compared with the treatment as usual group. Self-rated QoL was higher in the CST group as measured by the Quality of Life-AD. ${ }^{12}$ Last, there was a positive trend for communication (Holden Communication Scale). ${ }^{13}$ No significant differences were found for the secondary outcomes such as functional ability, anxiety, and depression.

The trial met some challenges and the research team made key observations that helped them to better understand the results. ${ }^{14}$ One of the limitations was the short follow-up period, which consisted of 8 weeks and did not allow for any evidence regarding long-term effects of CST. In addition, none of the staff-rated scales showed any significant benefits (behavior, mood, communication). The researchers did observe a considerable amount of variation between centers in terms of the effects on the outcome measures. This finding could be due to the role of staff members and the quality of the environment, but also to the level of impairment of participants. At times, if people with dementia were functioning quite well already, there was little room for significant improvement. It could also be difficult to run groups with people with different stages of dementia because those with mild impairment sometimes grew frustrated with participants with a greater degree of impairment. To maximize the effectiveness of the intervention, it is crucial to create an optimal learning environment, including pitching the sessions to an appropriate level according the needs of the group participants. Despite these challenges, the significant improvements on the primary outcome measures and the fact that people with dementia really enjoyed CST encouraged the research team to publish the CST training manual and to make it more widely available.

A few years later, the CST findings from the trial were supported with qualitative data when researchers investigated the experiences of people with dementia, carers, and group facilitators who attended CST groups. ${ }^{15}$ This study included 38 participants 
recruited from 3 existing CST groups. Two main themes (along with 7 subthemes) emerged from the focus groups and interviews: positive experiences of being in the group and changes experienced in everyday life. Participants shared many reflections, some of which are highlighted herein. Regarding changes in everyday life, participants reported noticing some benefits in their memory:

Yes, remembering the recent events have been a lot more simple and a lot more logical than it was certainly.

\section{- Person with dementia}

Cognitive benefits in other areas such as communication were also observed by carers.

She's clearer on the telephone. Clearer I suppose in the way she holds the conversation it's not that she speaks differently. It's just that the flow of the conversation is a little easier.

- Carer

Personal experiences reported by participants support the notion of CST being a positive and mentally stimulating experience, which is in line with previous quantitative findings. ${ }^{15}$

\section{MAINTENANCE COGNITIVE STIMULATION THERAPY}

The first CST trial showed positive results. However, the need for more research regarding potential longer term outcomes and more CST content for people with dementia in general, led to the development of an extended version of CST called maintenance CST (MCST). ${ }^{16}$ The MCST program includes the regular 7-week CST program with an extension of an additional 24 weekly maintenance sessions. Box 1 gives an overview of all the MCST themes in the published MCST manual.

Before finalizing the MCST program, an exploratory pilot study was conducted in 4 residential homes. ${ }^{17}$ After completion of the standard CST program, 2 residential homes were offered 16 once weekly MCST sessions and the 2 remaining homes served as treatment as usual control groups. Thirty-five participants were recruited for the study which were allocated to 1 of the 3 groups: (1) MCST and CST ( $n=8)$, (2) CST only ( $n=12)$, and (3) no CST $(n=15)$. Results indicated a continuous, significant improvement at follow-up on cognitive functioning as measured by the Mini Mental State Examination among participants receiving MCST (CST plus MCST) compared with the CST only or the no CST groups. No significant effects were found for QoL, communication, or behavior after MCST. It was evident that a fully developed MCST program was needed to formally investigate the effects of CST delivered over a longer term basis. ${ }^{17}$

The researchers considered the theory behind the original CST program and the findings from the exploratory pilot study while finalizing the MCST program. In line with CST, MCST was developed according to the Medical Research Council framework and used a mixed methods approach. ${ }^{16}$ Evidence from the following sources were combined: (1) a Cochrane review of cognitive stimulation for people with dementia, ${ }^{18}$ (2) a Delphi consensus process (involving key stakeholders), (3) focus groups with key stakeholders, and (4) a Delphi survey. This process led to the development of the MCST manual, which includes themed sessions and resembles the consistent structure of CST (eg, group name/song, noncognitive warmup). ${ }^{19}$ The finalized MCST program was evaluated in a large-scale randomized, controlled trial. 


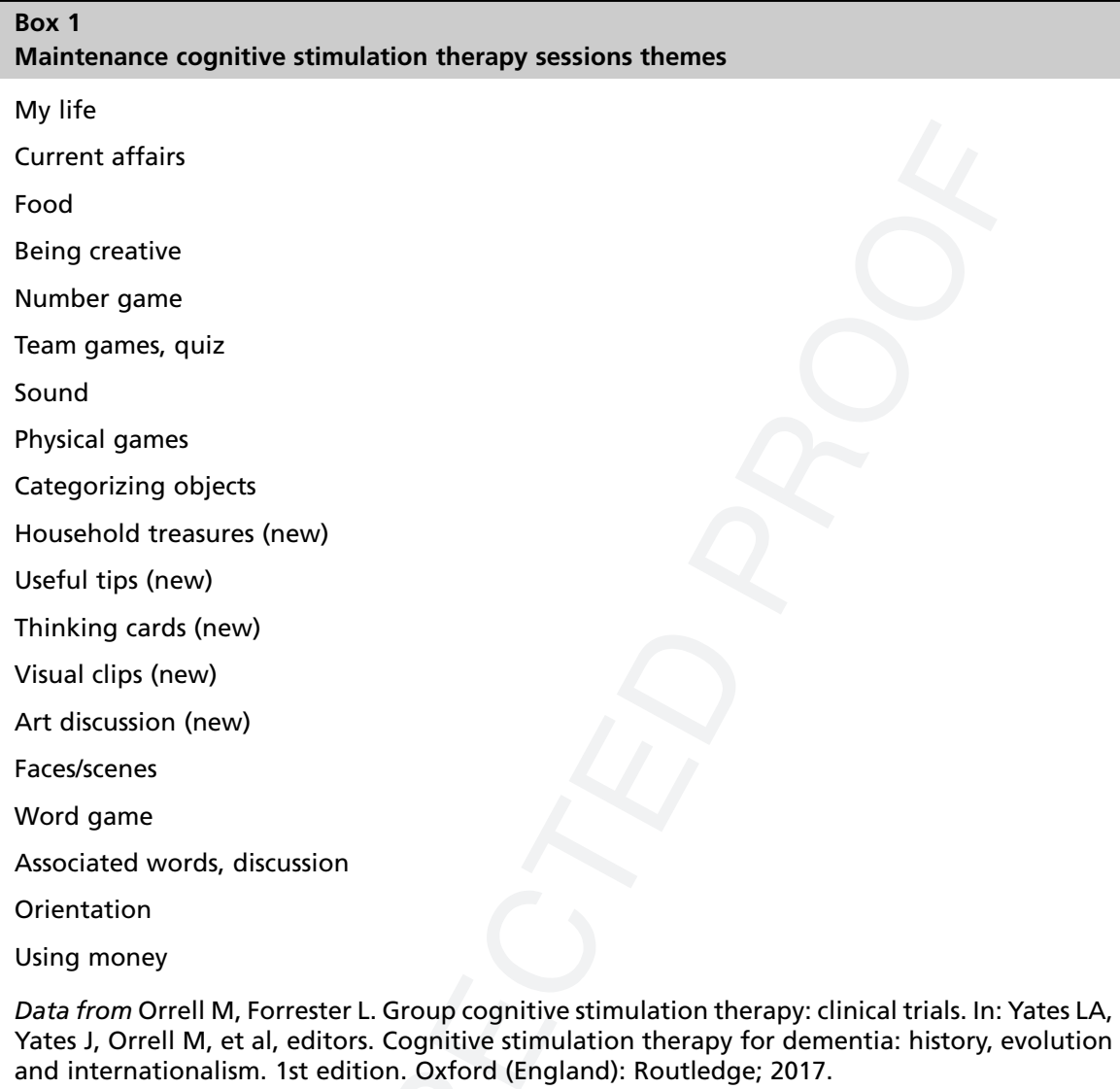

The MCST trial was a single-blind, multicenter, pragmatic randomized, controlled trial of the effects of MCST groups after the completion of the standard CST program versus CST followed by treatment as usual. ${ }^{20} \mathrm{~A}$ total of 236 participants were recruited from 9 care homes and 9 community services (eg, day centers). After completion of the original CST program, participants were randomly allocated to either the additional MCST program $(n=123)$ or the treatment as usual control group $(n=113)$. Participants were assessed at baseline before randomization had taken place, at 3 months, and after 6 months. Similarly, with the previous CST trial, the primary outcomes measures were the ADAS-Cog ${ }^{11}$ and QoL-AD. ${ }^{12}$

Trial results indicated that, at the 6-month follow-up, the MCST treatment group showed significant improvements in self-rated QoL-AD compared with the treatment as usual control group. ${ }^{21}$ At the 3-month follow-up, results showed positive effects for people with dementia on the proxy-rated QoL (Dementia Quality of Life questionnaire) ${ }^{22}$ by carers and care staff, and daily activities (Alzheimer's Disease Cooperative Study Activities of Daily Living Inventory). ${ }^{23}$ No significant effects were found on the ADAS-Cog or other secondary outcomes at either follow-up.

The most notable difference between the findings from the MCST trial and the first CST trial was the absence of improvements on cognition after MCST. ${ }^{21}$ Because dementia is associated with a progressive decline in cognition, participants in both the 
MCST and the control group were likely to have shown cognitive deterioration at the 6 month follow-up. This decline might have limited further cognitive improvement with MCST after the standard CST program. Another key finding came from a substudy of the MCST trial that investigated the use of acetylcholinesterase inhibitor medication in combination with MCST. The substudy found that less cognitive decline occurred in the MCST group taking acetylcholinesterase inhibitor medication compared with the MCST group without medication and the treatment as usual group. This finding indicates that better results might be obtained if pharmacologic treatments are combined with CST. The research team concluded that more research is needed regarding continued CST because this was the first rigorous trial of MCST and the results did not seem to be conclusive. However, because the significant improvements on QoL owing to MCST were an encouraging finding, the research team published the MCST manual.

\section{INDIVIDUAL COGNITIVE STIMULATION THERAPY}

With the increasing evidence for the benefits of CST and its uptake in routine services, the need to offer CST through different avenues became apparent. It was acknowledged that CST is not always accessible for those who are either unwilling or unable to attend groups. Taking their needs and wishes in consideration, the individual version of CST (iCST) was developed. Unlike CST and MCST, iCST is home based and is facilitated by an informal carer (eg, a family member, friend, or anyone who is close to the person with dementia) or a paid carer (eg, home support worker).

The development of iCST followed the Medical Research Council framework and included several research activities. ${ }^{24}$ In the first stages of development, people with dementia, carers, and care staff were asked to share their feedback and thoughts on the idea of iCST in an informal survey. The research team then reviewed existing literature of CST, MCST, one-to-one programs of cognitive stimulation, and RO. The evidence collated from the literature was then reviewed by a small group of key stakeholders such as carers and health care professionals who provided their advice on important considerations for the adaptation of CST to iCST. These activities led to the first draft of sessions 1 to 12 of the iCST manual, which were appraised in focus groups and interviews with people with dementia and carers. Participants were generally positive about the iCST materials and also shared their views on mentally stimulating activities and the feasibility of iCST. The research team proceeded with a field testing phase of the full program, which included both informal carers and paid carers. Both quantitative (eg, questionnaires, rating of enjoyment, interest, communication, and level of interest) and qualitative data (eg, through telephone support) were collected. Last, a 2-stage modified Delphi consensus process (online survey and conference) was used to reach consensus on themes that participants of focus groups, interviews, and field testing could not agree on. The sample consisted of academic, health care professionals, researchers, and carers. ${ }^{24,25}$

The iCST intervention follows the same principles of group CST; however, a few adjustments had to be made to make it suitable for use at home. Instead of the introduction and closing element of group CST, iCST sessions begin with a discussion of orientation information and current affairs followed by a themed activity. Each iCST session lasts around 20 to 30 minutes and each CST and MCST session was split to create 2 iCST sessions, which resulted in a 75-session program lasting over 25 weeks. Box 2 gives an overview of the iCST session themes; some themes occur more than once. The iCST omits the key principles geared toward the group process; 


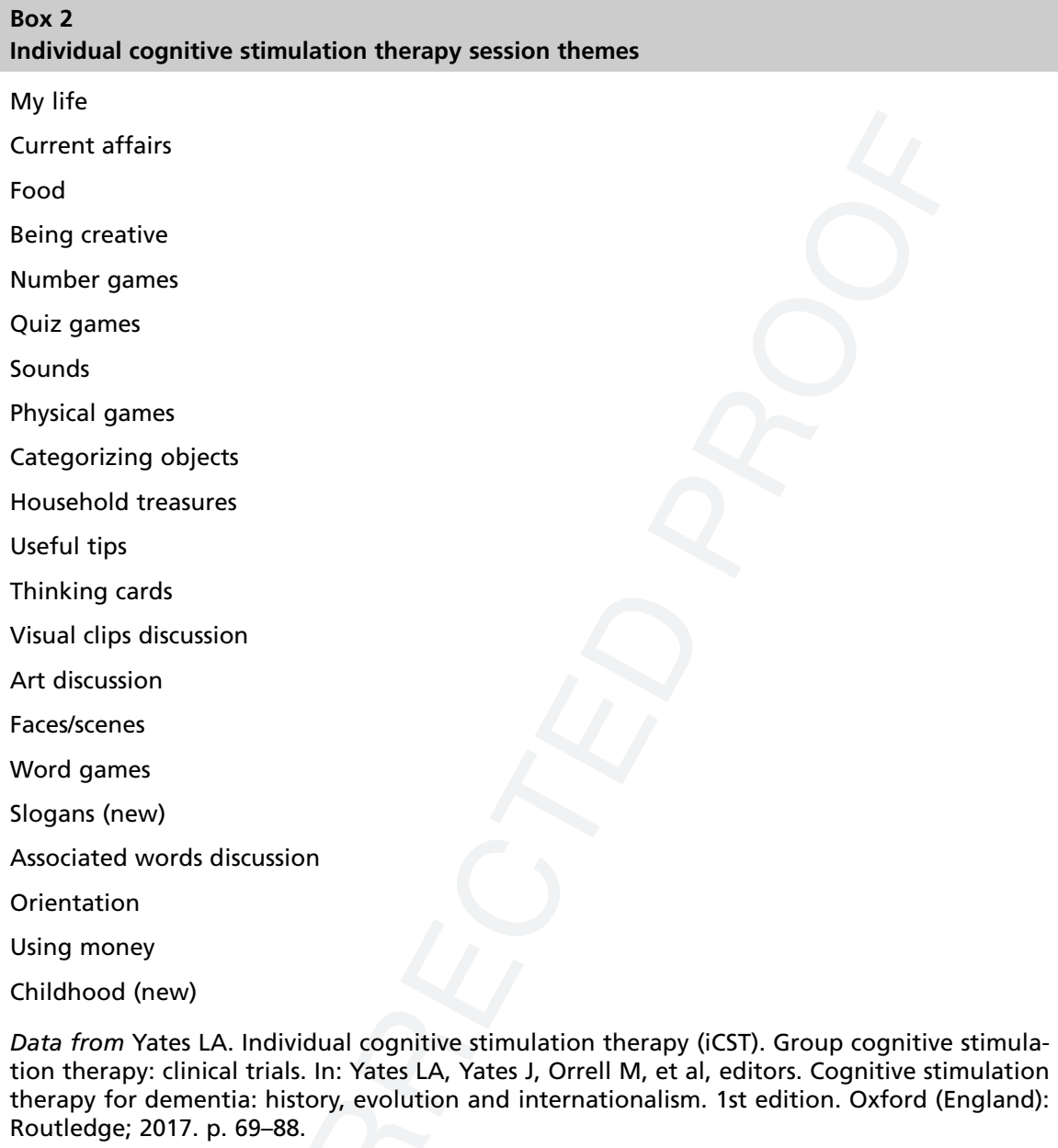

Data from Yates LA. Individual cognitive stimulation therapy (iCST). Group cognitive stimulation therapy: clinical trials. In: Yates LA, Yates J, Orrell M, et al, editors. Cognitive stimulation therapy for dementia: history, evolution and internationalism. 1st edition. Oxford (England): Routledge; 2017. p. 69-88.

rather, it stimulates discussion between the person with dementia and the carer and encourages them to enjoy the time they spend together.

The final iCST program was tested in a multicenter, single-blind, large-scale randomized, controlled trial. ${ }^{26}$ A total of 356 participants were recruited from a variety of community settings and allocated to either the iCST intervention group $(n=180)$ or the treatment as usual control group $(n=176)$. All participants met the Spector and colleagues (2003) standardized criteria with the addition of the following 2 criteria: living in the community and the availability of an informal carer. The main outcome measures were cognition (ADAS-Cog) ${ }^{11}$ and QoL for the person with dementia (QoL-AD), ${ }^{12}$ and QoL of the carer (Short Form-12). ${ }^{27}$ The primary and secondary outcomes measures were completed at 3 time points: baseline, first follow-up at 13 weeks, and second follow-up at 26 weeks. Throughout the trial, participants received support from the research team in the form of regular telephone support and monitoring visits. The trial results demonstrated no differences between the iCST and treatment as usual control group on any of the primary outcome measures at both follow-up time points. However, for one of the secondary outcome measures, 
significant improvements in the quality of the caregiving relationship from the person with dementia's perspective were found. For the carers, scores on a secondary QoL measure $(E Q-5 D)^{28}$ were significantly better in the iCST group at the second followup. $^{26}$

The results of this trial are not consistent with previous CST findings and the following reflections may help us to better understand the iCST evidence. Because the iCST is a longer intervention, the findings might indicate that a short-term, more intense dose of CST could be more beneficial or effective. The social setting provided during the group CST might also be crucial to enhancing cognition and QoL; thus, lacking this feature, the iCST may not elicit benefits. It is suggested, in previous research, that improvements in cognition from the CST mediate improvements in QoL for people with dementia. ${ }^{18}$ Hence, the lack of change in cognition experienced by iCST participants could explain the lack of results on QoL. The greatest challenge of the trial proved to be adherence to iCST. The research team observed that, on average, dyads completed just less than one-half of the recommended 75 sessions over 25 weeks. Although, before the trial, during the development phase, carers determined the current iCST format to be feasible, in reality carers identified several barriers to delivering the intervention after the trial, such as time constraints, physical health problems, and motivation.

Despite the lack of significant effects on cognition and QoL, this trial was innovative for several reasons. The iCST trial is the largest known piece of CST research to date and it is the first trial investigating a home-based, carer-led format of CST. This trial demonstrated that, in general, carers are able to deliver an intervention, which is a key finding supporting carer-led interventions. The observed improvements in the quality of the caregiving relationship are encouraging and could enhance the QoL of people with dementia. The results from this trial are not conclusive and there is a need for continued research on iCST to determine its exact effectiveness.

\section{INTERNATIONAL COGNITIVE STIMULATION THERAPY}

CST was initially developed and implemented in the UK and, after its success, began to attract international attention. Given the cultural differences in almost every country, in addition to the language barriers, it was deemed crucial to have some kind of framework in place that could facilitate the adaptation of CST. Therefore, the research team at UCL set out to create guidelines that could inform the process of adapting and translating the CST content and structure without compromising on its effectiveness. $^{29}$

The research team reviewed existing frameworks and theoretic methods that have been developed to guide the cultural adaptation of existing interventions. Of the frameworks reviewed, the formative method for adapting psychotherapy was chosen to develop the CST guidelines owing to its community-based developmental approach. ${ }^{30}$ This is a bottom-up approach in which people with dementia and other service users are consulted as a preliminary step to uncover their ideas and opinions (eg, how dementia is perceived in their culture). This step is essential because it provides an early understanding of how CST can be catered toward the needs of service users in that specific country. The formative method for adapting psychotherapy approach together with evidence from existing international CST groups resulted in guidelines consisting of 5 phases, which are described in Fig. 1.

Currently, CST is used in all of the following developed and developing countries: Australia, Brazil, Canada, Chile, China, Denmark, Germany, Greece, Hong Kong, India, Israel, Italy, Ireland, Indonesia, Japan, Nepal, the Netherlands, New Zealand, 


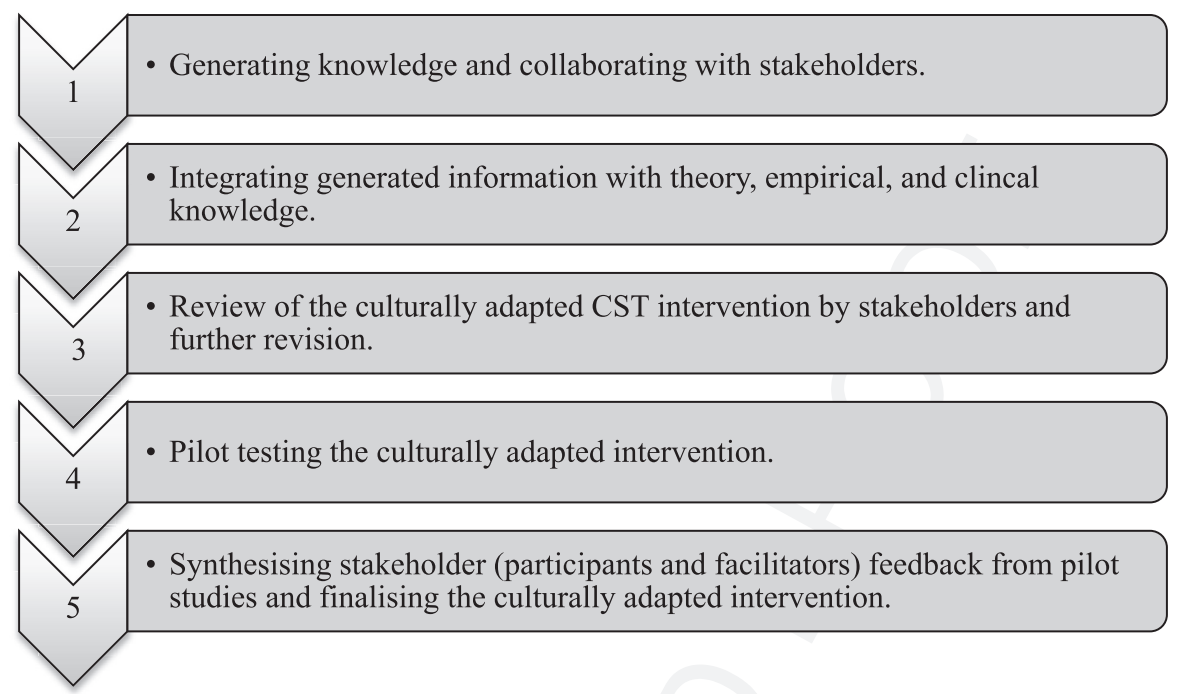

Fig. 1. The 5 phases of cognitive stimulation therapy adaptation guidelines.

Nigeria, Philippines, Portugal, Singapore, South Africa, South Korea, Tanzania, Turkey, and the United States. ${ }^{31}$ CST is especially relevant for developing countries because it provides an effective low-cost intervention to help improve cognition and QoL. Establishing the international CST center at UCL has been a crucial step toward making CST more widely available because the center has been facilitating collaborations and knowledge exchange between the more the 25 countries currently offering CST.

\section{COGNITIVE STIMULATION THERAPY IN THE UNITED STATES: A CASE STUDY}

CST has been successfully adapted and implemented in the United States. ${ }^{32}$ The first comprehensive CST program was developed at the Perry County Memorial Hospital, a small rural hospital in southeastern Missouri. Before its development, professionals received visits and CST training from members of the UCL research team. CST was found to be easily adaptable for the US population because the language barrier was minimal and sessions that focused on universal topics such as faces and scenes or food allowed for cultural adaptation. ${ }^{32}$ After the adaptation of group CST, researchers compared pre-CST and post-CST data among both community-based and residential-dwelling people with dementia $(n=79)$ in which they found scores for cognition and QoL to be higher after the CST. These findings have been encouraging and allowed for the expansion of CST at Perry County Memorial Hospital from one, 6-member CST group to 10 CST groups currently running every week catering for 90 people with dementia. With regard to iCST, a family carer (daughter of a person with dementia) evaluated it quite positively as illustrated by the following quote:

From discussion they had over architecture to deep thoughts on top news stories to exploring timeless paintings to simply relishing in discussing the glorious days of old, dad lover every moment. These weekly visits continued throughout dad's stay in the hospital and extended to his home post discharge. Dad would continue on in thought and articulate communication. Their session bled over into the everyday. And over time, dad became increasingly more fluent and lucid. 
The US research team continues to provide CST training around the country and plans to establish a US CST National Training Center are underway. The team hopes to publish an adapted CST manual in the near future.

\section{SUMMARY AND FUTURE DIRECTIONS}

The CST journey has spanned for more than 20 years so far and innovations continue to be made in this field. When CST was developed, it helped to fill the existing gap in evidence-based psychological treatments for people with dementia. In this regard, it can be seen as a fundamental step toward shifting some of the focus from pharmacologic treatments to psychological ones. The positive effects of CST further amplified the importance of looking beyond antidementia medication and it fueled the realization that the two might actually provide the most optimal benefits to people with dementia when combined. Findings regarding experiences of people with dementia were just as encouraging; people have reported enjoyment and even increased confidence after CST. Therefore, CST has managed to provide both a meaningful and stimulating way for people with dementia to spend their time. The success of the original group CST made it possible to go even further and develop extensions of group CST ensuring that the intervention can be offered to people with different needs. In addition, the adaptation guidelines made it possible for CST to successfully be adapted and offered in a variety of countries around the world.

Still, there is more to be explored in the field of CST because some questions remain unanswered. The optimal dose for long-term CST is unknown and future research could help to give an indication of what the most beneficial duration and frequency of CST could be. Other work could focus on experimenting with iCST; for example, enhancing methods of support and training could help to improve adherence. In terms of exploring different platforms for CST, incorporating technology seems to be an attractive option; the use of technology can benefit the cognitive functioning of older people. A pilot study in Italy has investigated a tablet-based version of CST that can be delivered at home. ${ }^{33}$ Results suggest the need for more research in this area.

For the future, we hope to maintain this growth of CST and explore different avenues for offering CST on both a national and international level. We aim to continue connecting stakeholders from around the world at our CST conferences and generate ideas and discussions on what works and what can be done even better. This would help to create an even better understanding of CST and encourage other researchers and clinicians to explore the field of CST so that CST will continue to be available to people with dementia who want and need it.

\section{REFERENCES}

1. Hodge S, Hailey E, Orrell M, editors. Memory services national accreditation programme - standards for memory services. 4th edition. London: Royal College of Psychiatrists; 2014.

2. Knapp M, Spector A, Thorgrimsen L, et al. Cognitive Stimulation Therapy for people with dementia: cost effectiveness analysis. Br J Psychiatry 2006;188:574-80.

3. Orrell M, Woods B. Editorial comment. Tacrine and psychological therapies in dementia - no contest? Int J Geriatr Psychiatry 1996;11(3):189-92.

4. Breuil V, De Rotrou J, Forette F, et al. Cognitive stimulation of patients with dementia. Int J Geriatr Psychiatry 1994;9(3):211-7.

5. Spector A, Thorgrimsen L, Woods B, et al. Making a difference: an evidence based group program to offer Cognitive Stimulation Therapy (CST) to people with dementia. London: Hawker Publications; 2006. 
6. Craig P, Dieppe P, Macintyre S, et al. Developing and evaluating complex interventions: new guidance. Medical Research Council 2006.

7. Spector A, Orrell M, Davies S, et al. Can reality orientation be rehabilitated? Development and piloting of an evidence-based programme of cognitionbased therapies for people with dementia. Neuropsychol Rehabil 2001;11(3/4): 377-97.

8. Spector A, Thorgrimsen L, Woods B, et al. Efficacy of an evidence-based cognitive stimulation therapy programme for people with dementia: randomised controlled trial. Br J Psychiatry 2003;183:248-54.

9. American Psychiatric Association. Diagnostic and statistical manual of mental health disorders. 4th edition. Washington, DC: APA; 1994.

10. Folstein MF, Folstein SE, McHugh PR. "Mini mental state". A practical method for grading the cognitive state of patients for the clinician. J Psychiatr Res 1975; 12(3):189-98.

11. Rosen WG, Mohs RC, Davis KL. A new rating scale for Alzheimer's Disease. Am J Psychiatry 1984;141(11):1356-64.

12. Logsdon RG, Gibbons LE, McCurry SM, et al. Quality of life in Alzheimer's disease: patient and caregiver reports. J Ment Health Aging 1999;5:21-32.

13. Holden UP, Woods RT. Positive approaches to dementia care. 3rd edition. Edinburgh (Scotland): Churchill-Livingstone; 1995.

14. Orrell M, Forrester L. Group cognitive stimulation therapy: clinical trials. In: Yates LA, Yates J, Orrell M, et al, editors. Cognitive stimulation therapy for dementia: history, evolution and internationalism. 1st edition. Oxford (England): Routledge; 2017. p. 49-67.

15. Spector A, Gardner C, Orrell M. The impact of Cognitive Stimulation Therapy groups on people with dementia: views from participants, their carers and group facilitators. Aging Ment Health 2011;15(8):945-9.

16. Aguirre E, Spector A, Hoe J, et al. Development of an evidence-based extended programme of maintenance Cognitive Stimulation Therapy (CST) for people with dementia. Non-pharmacological Therapies in Dementia Journal 2011;1:198-215. a10

17. Orrell M, Spector A, Thorgrimsen L, et al. A pilot study examining the effectiveness of Maintenance Cognitive Stimulation Therapy (Maintenance CST) for people with dementia. Int J Geriatr Psychiatry 2005;11:446-51.

18. Woods B, Aguirre E, Spector A, et al. Cognitive stimulation to improve cognitive functioning in people with dementia. Cochrane Database Syst Rev 2012;(2):CD005562.

19. Aguirre E, Spector A, Streater A, et al. Making a Difference 2: an evidence based group program to offer maintenance Cognitive Stimulation Therapy (CST) to people with dementia. London: Hawker Publications; 2006.

20. Aguirre E, Hoare Z, Streater A, et al. Cognitive Stimulation Therapy (CST) for people with dementia - who benefits most? Int J Geriatr Psychiatry 2013;28(3): 284-90.

21. Orrell M, Aguirre E, Spector A, et al. Maintenance Cognitive Stimulation Therapy (CST) for dementia: a single-blind, multi-centre, randomised controlled trial of Maintenance CST vs. CST for dementia. Br J Psychiatry 2014;204:454-61.

22. Smith SC, Lamping DL, Banerjee S, et al. Measurement of health-related quality of life for people with dementia: development of a new instrument (DEMQOL) and an evaluation of current methodology. Health Technol Assess 2005;9(10).

23. Galasko D, Bennet D, Sano M, et al. An inventory to assess activities of daily living for clinical trials in Alzheimer's disease: the Alzheimer Disease Cooperative Study. Alzheimer Dis Assoc Disord 1997;11:S33-9. 
610

611

612

613

614

615

616

617

618

619

620

621

622

623

624

625

626

627

628

629

630

631

632

633

634

635

636

637

638

639

640

641

24. Yates LA, Leung P, Orgeta V, et al. The development of individual cognitive stimulation therapy (iCST) for dementia. Clin Interv Aging 2015;10:95-104.

25. Yates L, Orrell M, Leung $P$, et al. Making a difference 3 individual CST: a manual for carers. London: Hawker Publications; 2014.

26. Orrell $M$, Yates $L$, Leung $P$, et al. The impact of individual Cognitive Stimulation Therapy (iCST) on cognition, quality of life, caregiver health, and family relationships in dementia: a randomised controlled trial. PLoS Med 2017;14(3):1-22.

27. Ware J Jr, Kosinski M, Keller SD. A 12-Item short form health survey: construction of scales and preliminary tests of reliability and validity. Med Care 1996;34: 220-33.

28. EuroQoL Group. EuroQoL-a new facility for the measurement of health-related quality of life. Health Policy 1990;16:199-208.

29. Aguirre E, Werheid K. Guidelines for adapting cognitive stimulation therapy to other cultures. In: Yates LA, Yates J, Orrell M, et al, editors. Cognitive stimulation therapy for dementia: history, evolution and internationalism. 1st edition. Oxford (England): Routledge; 2017. p. 177-93.

30. Hwang WC. The formative method for adapting psychotherapy (FMAP): a community-based developmental approach to culturally adapting therapy. Prof Psychol Res Pr 2009;40(4):369.

31. International CST Groups. 2018. CST Dementia website Available at: http://www. cstdementia.com. Accessed January 24, 2018.

32. Lundy L, Hayden D, Berg-Weger M, et al. United States. In: Yates LA, Yates J, Orrell $\mathrm{M}$, et al, editors. Cognitive stimulation therapy for dementia: history, evolution and internationalism. 1st edition. Oxford (England): Routledge; 2017. p. 215-26.

33. Gardini S, Michelini G, Tirelli P, Morciano E, Caffarra P. The development of a home-based and computerized Cognitive Stimulation Therapy for people living with dementia: preliminary results. In: Proceedings from the Second International Meeting of the Milan Center for Neuroscience (Neuromi): Prediction and Prevention of Dementia: New Hope; July 6-8, 2016; Milan, Italy. S24. 


\section{AUTHOR QUERY FORM}

\begin{tabular}{|c|c|}
\hline 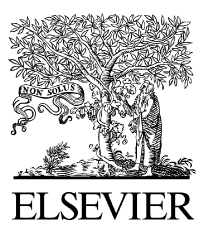 & $\begin{array}{l}\text { Journal: CGM } \\
\text { Article Number: } 903\end{array}$ \\
\hline
\end{tabular}

Dear Author,

Please check your proof carefully and mark all corrections at the appropriate place in the proof (e.g., by using on-screen annotation in the PDF file) or compile them in a separate list. It is crucial that you NOT make direct edits to the PDF using the editing tools as doing so could lead us to overlook your desired changes. Note: if you opt to annotate the file with software other than Adobe Reader then please also highlight the appropriate place in the PDF file. To ensure fast publication of your paper please return your corrections within 48 hours.

For correction or revision of any artwork, please consult http://www.elsevier.com/artworkinstructions.

Any queries or remarks that have arisen during the processing of your manuscript are listed below and highlighted by flags in the proof.

\begin{tabular}{|c|c|}
\hline $\begin{array}{l}\text { Location } \\
\text { in article }\end{array}$ & $\begin{array}{l}\text { Query / Remark: Click on the } Q \text { link to find the query's location in text } \\
\text { Please insert your reply or correction at the corresponding line in the proof }\end{array}$ \\
\hline Q1 & Please approve the short title to be used in the running head at the top of each right-hand page. \\
\hline Q2 & Are author names and order of authors OK as set? \\
\hline Q3 & $\begin{array}{l}\text { This is how your name will appear on the contributor's list. Please add your academic title and any other } \\
\text { necessary titles and professional affiliations, verify the information, and OK } \\
\text { HARLEEN RAI, MSc, Division of Psychiatry and Applied Psychology, Institute of Mental Health, School } \\
\text { of Medicine, University of Nottingham, Jubilee Campus, Nottingham, United Kingdom } \\
\text { LAUREN YATES, BSc, PhD, Division of Psychiatry and Applied Psychology, Director, Head, Institute of } \\
\text { Mental Health, School of Medicine, University of Nottingham, Jubilee Campus, University of Nottingham } \\
\text { Innovation Park, Nottingham, United Kingdom } \\
\text { MARTIN ORRELL, PhD, FRCPsych, Division of Psychiatry and Applied Psychology, Faculty of } \\
\text { Medicine and Health Sciences, Research Fellow, Institute of Mental Health, University of Nottingham, } \\
\text { Jubilee Campus, University of Nottingham Innovation Park, Nottingham, United Kingdom }\end{array}$ \\
\hline Q4 & $\begin{array}{l}\text { The following synopsis is the one that you supplied, but lightly copyedited. Please confirm OK. Please note } \\
\text { that the synopsis will appear in PubMed: Cognitive stimulation therapy has proven to be both an effective } \\
\text { and enjoyable psychological treatment for people with dementia. Over the past } 20 \text { years, cognitive } \\
\text { stimulation therapy has grown from a national, localized treatment in the UK to a more global phenomenon } \\
\text { currently being used in more than } 25 \text { countries around the world. Much has been accomplished during the } \\
\text { cognitive stimulation therapy journey and there is still much to be explored; it is a dynamic field. This } \\
\text { article provides an overview of cognitive stimulation therapy by elaborating on its background, evidence, } \\
\text { international work, and future directions. }\end{array}$ \\
\hline Q5 & Please verify the affiliation addresses. \\
\hline Q6 & $\begin{array}{l}\text { If there are any drug dosages in your article, please verify them and indicate that you have done so by } \\
\text { initialing this query. }\end{array}$ \\
\hline
\end{tabular}




\begin{tabular}{|l|l|} 
Q7 & $\begin{array}{l}\text { Nominal tables that are really just one-column lists are best represented as boxes, so Tables } 2 \text { and } 3 \text { have } \\
\text { been converted to Boxes } 1 \text { and } 2 \text { respectively. Please verify. } \\
\text { The journal title in Ref. } 6 \text { does not match that in PubMed. Please check that the reference details are correct } \\
\text { and also provide the volume number or issue number and page range. } \\
\text { Please verify the edits made in publisher location of Refs. 14, 29, 32. } \\
\text { Q10 }\end{array}$ \\
Q11 & $\begin{array}{l}\text { The journal title in Ref. 16 does not match that in PubMed. Please check that the reference details are } \\
\text { Please clarify the publication type (e.g., proceedings, journal, or book) in Ref. } 33 . \\
\text { Please provide page range for source line in Box 1. }\end{array}$ \\
$\begin{array}{l}\text { Please check this box or indicate } \\
\text { your approval if you have no } \\
\text { corrections to make to the PDF file }\end{array}$
\end{tabular}

Thank you for your assistance. 